\title{
Evaluation Profile In Vitro Release Gastroretentive High Density Tablet Theophylline Using Sodium Alginate and PVP
}

\author{
Amran Nur $^{1 *}$, Ermalyanti Fiskia ${ }^{1}$, and Bambang Tjiroso ${ }^{2}$ \\ ${ }^{1}$ Departement of Pharmacy, Medical Faculty, Khairun University, 97719 South Moluccas, Indonesia \\ ${ }^{2}$ Departement of Mechanical Engineering, Engineering Faculty, Khairun University, 97719 South Moluccas, Indonesia
}

\begin{abstract}
The high-density gastroretentive dosage forms was made a high-density theophylline tablets 250 $\mathrm{mg}$ by wet granulation method in three formulas with combine the polymer concentration. The polymer used sodium alginate and PVP K30, where the 1st formula ratio of sodium alginate: PVP K30 (18.75\%: 5\%), the 2nd formula ratio of sodium alginate: PVP K30 (18.75\%: 2, 5\%) and 3th formula ratio of sodium alginate: PVP K30 (12.5\%: 2.5\%). The third formula were evaluated with weight uniformity test, size uniformity test, hardness test, drug content test, in vitro release test, as well as the determination of the kinetics of drug release. The result showed the uniformity weights of the formula ranges $250.12 \mathrm{mg}-250.70 \mathrm{mg}$, the uniformity size of the formula ranges $0.81 \mathrm{~cm}$ for diameter tablet and $0.42 \mathrm{~cm}-0.44 \mathrm{~cm}$ for thick tablet, the hardness test ranges from $90 \mathrm{~N}-180 \mathrm{~N}$. Results obtained for drug content was $106.965 \%$ formula 1 st, formula 2nd is $127.625 \%$ and for formula 3 th is $115.976 \%$. Release kinetic profiles show formula 1 st and 2nd used Higuchi equation and formula 3th used the first-order equation. By these three formula, 1st formula was considered the best views of drug content and release kinetics.
\end{abstract}

Keywords: Gastroprotective, High Density, GRDDS, Theophylline, Sodium Alginate, PVP K30

\section{Introduction}

Drugs is a chemical substance, typically of known structure. Drug used for diagnosis, reducing pain, and treating or preventing disease in humans[1]. Drugs are an important element in the health sector, most medical interventions use drugs, therefore drugs must be available in sufficient quantities, real efficacious and of good quality[2]

Drugs can be given by oral, parenteral, rectal and topical routes[1]. The oral route is the most popular and convenient route for various drugs. The oral route is generally considered an ideal drug delivery system which will have two main properties namely it must be in a single dose to prolong the action and must deliver the active drug ingredients directly to the target site[3].

In conventional oral drug delivery systems there is no control over drug release and effective concentration of overdosage[4] changing dosage patterns can cause side effects in some cases[5]. Therefore the rate and extent of drug absorption from conventional drug formulations can be different, depend on the factors such as the physicochemical properties of the drug, the presence of additives and various physiological factors such as the presence or absence of food and the $\mathrm{pH}$ of the gastrointestinal system[6]

In order to avoid many of the potential problems associated with the use of conventional dosage forms, the concept of controlled release has been applied for the development of new dosage formulations, a number of approaches have been used to increase the retention time in the stomach of the dosage forms in the stomach using a variety of concepts[7]. One form of drug delivery that produces a slow-release action is the high-density system in which the drug is retained in the lower part of the stomach for long periods of time[7]

The high density system is a system that the drug can be trapped in the folds of the antrum and resist peristaltic waves from the stomach wall[8][9] Theophylline is one of the drugs that can be made in the form of high density gastroretentive dosage forms because it is thought to be able to increase bioavailability in the body[10].

Theophylline is still one of the most widely prescribed drugs for the treatment of asthma and chronic obstructive pulmonary disease (COPD) worldwide, because it is inexpensive and widely available. Theophylline (dimethylxanthine) occurs naturally in tea and cocoa beans in trace amounts. Theophylline has a relatively short half-life and a narrow therapeutic index of $5-20 \mathrm{~g} / \mathrm{ml}$. The sustained-release formulation is expected to produce a more uniform blood concentration of the drug, with a peak level that does not fluctuate. Slow-release dosage forms can guarantee patient satisfaction, especially if the patient has difficulty taking the drug repeatedly during an acute asthma attack [11]

A polymer is needed to control the drug release system[12], a hydrophilic polymer is widely used in the formulation of oral sustained release dosage forms [13]. 
One of the hydrophilic polymers that can be used in sustained release dosage forms is sodium alginate. and PVP, where sodium alginate will form a gel layer in the form of alginic acid at an acidic $\mathrm{pH}$ (gastric environment) [14] and PVP which functions to release the drug rapidly from the matrix resulting in a slow release from the expanded matrix [15].

\section{Materials and Methods}

\subsection{Material}

Aquadest, 96\% Alcohol, 0.1 N HCl Solution, Lactose, Sodium Alginate, Sodium Bicarbonate, Magnesium Stearate, Talc, Theophylline and Polyvinylpyrrolidone

\subsection{Preparation of Tablets}

Tablets are prepared by wet granulation method with heat. Theophylline is put into a mortar then polymer is added, then sodium bicarbonate and lactose are added and then mix until homogeneous. The homogeneous mixture was then sieved using a sieve with a mesh number of 60 and aquadest was added little by little until a moist mass was formed. The moist mass was then sieved using a sieve with mesh no. 10 and dried in an oven at $50^{\circ} \mathrm{C}$ for 12 hours. The dried granules were then sieved using a sieve with a mesh number of 16 , the sifted granules were then added to the lubricant and then compressed using a tablet press.

Table 1. Formula design of high density theophylline gastroretentive tablets

\begin{tabular}{|l|c|c|c|}
\hline \multicolumn{1}{|c|}{ Materials } & F1 & F2 & F3 \\
\hline Theophylline (mg) & 109,37 & 109,37 & 109,37 \\
\hline Sodium Alginate (mg) & 46,87 & 46,87 & 31,25 \\
\hline $\begin{array}{l}\text { Polyvinylpyrrolidone } \\
\text { (mg) }\end{array}$ & 12,50 & 6,25 & 6,25 \\
\hline Sodium Bicarbonat (\%) & 1 & 1 & 1 \\
\hline Talc (\%) & 2 & 2 & 2 \\
\hline Magnesium Stearate(\%) & 1 & 1 & 1 \\
\hline Lactosa add 250 mg & 71,26 & 77,51 & 93,13 \\
\hline
\end{tabular}

\subsection{Evaluation of tablet properties}

Tablets were evaluated as per the following tests:

\subsubsection{Uniformity of weight}

Twenty (20) randomly sampled tablets were weighed individually on a precision balance and the average determined.

\subsubsection{Crushing strength}

Tablet crushing strength (CS) or hardness which is the force required to diametrically cause a tablet to fracture was determined using a Lab. Hosp. hardness tester. The test was repeated twice and the mean recorded.

\subsubsection{Drug content test}

One tablet of each formula was taken and then ground until smooth and then sonicated in $100 \mathrm{~mL}$ of $0.1 \mathrm{~N} \mathrm{HCl}$ solution $\mathrm{pH} 1.2$ for 5 minutes then the absorbance was measured on a UV-Vis spectrophotometer at a wavelength of $269.45 \mathrm{~nm}$ which was obtained from the determination of the wavelength. maximum wave.

\subsubsection{In vitro drug dissolution studies}

One tablet was inserted into the USP type II dissolution apparatus (paddle) at a speed of $50 \mathrm{rpm}$ in $900 \mathrm{~mL}$ of 0.1 $\mathrm{N} \mathrm{HCl}(\mathrm{pH} 1.2)$ at a temperature of $37 \pm 0.5^{\circ} \mathrm{C}$. The test was carried out for 12 hours with an interval of every 60 minutes. Each $5.0 \mathrm{~mL}$ of sampling, was replaced with a new medium with a volume of $5.0 \mathrm{~mL}$. The test for each formula was repeated three times. The sample obtained was diluted by pipetting $0.8 \mathrm{~mL}$ of the sample using a micropipette and then the volume was made up to $10 \mathrm{~mL}$ and then the absorbance was measured on a UV-Vis spectrophotometer at a wavelength of $269.45 \mathrm{~nm}$ which was obtained from the determination of the maximum wavelength.

\section{Discussion}

The gastroretentive drug delivery system (GRDDS) dosage form consists of several types, one of which is a high density dosage form where the drug is retained in the lower part of the stomach for a long period of time. The high density system is a system with a high density so that the drug can be trapped in the folds of the antrum and resist peristaltic waves from the stomach wall.

One of the drug candidates that can be made in gastroretentive dosage forms is theophylline, where theophylline is a methyl xanthine derivative that functions in the treatment of bronchial asthma and bronchospasm reactions, has a short half-life and narrow therapeutic index.

Based on the results of the density test of the gastroretentive granules, the density values for formula 1 were $1.16 \mathrm{~g} / \mathrm{mL}$, formula 2 was $1.19 \mathrm{~g} / \mathrm{mL}$ and formula 3 was $1.24 \mathrm{~g} / \mathrm{mL}$. Seeing that the density obtained exceeds the density of gastric fluid, which is $\sim 1.004$ $\mathrm{g} / \mathrm{mL}$, in this study, high density theophylline tablets with a weight of $250 \mathrm{mg}$ were made in three formulas with a combination of polymer concentrations. The polymer used is sodium alginate and PVP, where in formula I the ratio of the polymer material is sodium alginate: PVP (18.75\%: 5\%), formula II the ratio of polymer material is sodium alginate: PVP (18.75\%: $2.5 \%$ ) and formula III the ratio of polymeric materials sodium alginate: PVP (12.5\%: $2.5 \%)$. 
Alginate and PVP are hydrophilic polymers and are used as a matrix in the formulation of sustained release tablets because they have good compressive properties. In addition, the hydrophilic polymer also has a fairly good swelling property which causes the formation of an inner layer in a fast time so that the drug can be released.

The formula is made by wet granulation method, where water is used as a granulating agent, this is based on the fact that the wet granulation method can provide good granule flow during the tablet compression process, besides theophylline which is used as the active ingredient can be stable against water and heat.

From the tablet weight uniformity test the average value for $\mathrm{F} 1$ is $250.50 \mathrm{mg}$ with a variance value of 19.327 based on the variance value, so formula 1 has a weight deviation of 4.396 against the average value, this value is known as the standard deviation. The average value for F2 is $250.12 \mathrm{mg}$ with a variance value of 21.427327 based on the variance value, so formula 2 has a weight deviation of 4.629 from the average value. The average value for F3 is $250.70 \mathrm{mg}$ with a variance value of 15.155 and based on the variance value, formula 3 has a weight deviation of 3.893 from the average value. Based on the requirements set by the Indonesian Pharmacopoeia edition III that the average deviation for tablets weighing $151 \mathrm{mg}-300 \mathrm{mg}$ for 20 tablets should not be more than two tablets whose weights deviate $7.5 \%$ from the average weight and there should not be one tablet that the weight deviates $15 \%$ from the average weight. The results obtained indicate uniformity for each tablet and also indicate the weight of the substance contained in the tablet is uniform in other words the dose for each tablet is stated to be uniform or the same.

For uniformity of size, 10 tablets of each formula were taken and then determined by measuring the diameter and thickness of the tablets using a caliper. From the measurement results, the average diameter for F1 is $0.81 \mathrm{~cm}$ with a variance value of $2.2 \times 10^{-5}$, then from the variance value obtained a standard deviation of 0.004 , and the average tablet thickness for $F 1$ is $0.44 \mathrm{~cm}$ with a variance value of $2.2 \times 10^{-5}$ and the standard deviation value is 0.004 . For F2 the average value of the diameter is $0.81 \mathrm{~cm}$ with a variance value of $4 \times 10^{-5}$ and a standard deviation value of 0.006 , the average tablet thickness for $\mathrm{F} 2$ is $0.42 \mathrm{~cm}$ with a variance value of $3.3 \times 10^{-5}$ and a standard deviation value of 0.005 . And for $\mathrm{F} 3$ the average tablet diameter is $0.81 \mathrm{~cm}$ with a variance value of $1.1 \times 10^{-5}$ and a standard deviation value of 0.003 , the average thickness of the F3 tablet is $0.43 \mathrm{~cm}$ with a variance value of $1.1 \times 10^{-4}$ with a standard deviation of 0.010 . Based on the requirements in the pharmacopoeia, the three tablet formulas meet the requirements for size uniformity, namely those with a diameter of not more than 3 times and not less than $4 / 3$ times the thickness of the tablet.

Hardness test is carried out by taking 10 tablets from each batch, which is then measured for hardness using a hardness tester. From the research results, the highest level of hardness in F1 is $180 \mathrm{~N}$ and the lowest value is $90 \mathrm{~N}$, with an average value of $152 \mathrm{~N}$, a variance value of 1217.77 and a standard deviation of 34.89 . In F2 the highest hardness level is $180 \mathrm{~N}$ and the lowest value is $150 \mathrm{~N}$ with an average value of $169 \mathrm{~N}$, a variance value of 98.99 and a standard deviation of 9.94. In F3 the highest hardness level is $180 \mathrm{~N}$ and the lowest value is $160 \mathrm{~N}$ with an average value of $170 \mathrm{~N}$, a variance value of 44.44 and a standard deviation of 6.67. From these results, it can be concluded that the high density theophylline gastroretentive tablet used in the test was only formula 3 which met the requirements for the hardness test based on the requirements that it did not exceed $20 \mathrm{~kg}(198 \mathrm{~N})$ and had the smallest standard deviation value when compared to the two previous formulas. while formula 1 and formula 2 do not meet the requirements, this is because both formulas have large standard deviation values based on statistical calculations which indicate that the drug hardness in one batch is not uniform.

Drug content test is carried out to determine the level of drug in the preparation to match the desired dose. The results obtained for formula 1 are $106.965 \%$, formula 2 is $127.625 \%$ and formula 3 is $115.976 \%$ (see table 5), Banker \& Rhodes (2009) stated that the drug content ranged from $85-115 \%$ of what is stated on the label, which is means that the formulas containing the drug content that meet the requirements are formula 1 and formula 3 while formula 2 does not meet the requirements this is due to the possibility that the tablets used in the test do not have a good distribution of drug content which arise due to problems when mixing and compressing the tablets.

In vitro drug release using one tablet was carried out for 12 hours in $0.1 \mathrm{~N} \mathrm{HCl}$ medium $\mathrm{pH} 1.2 \pm 0.1$ with an interval of every 60 minutes to determine how much drug was released per unit time. The test was carried out for 12 hours adjusted to the administration of a modified release form of theophylline 2 times a day, $\mathrm{HCl} \mathrm{pH} 1.2$ was used as a substitute for gastric fluid. The results showed that the cumulative release percentage for formula 1 was $90.83 \%$, for formula 2 as much as $99.48 \%$ and for formula 4 as much as $99.76 \%$ which means that almost all the drugs in the tablet were released at 12 hours.

\section{Conclusion}

Based on the results of tablet evaluation, the best formula is formula 3 with a ratio of sodium alginate: PVP is $31.25 \mathrm{mg}: 6.25 \mathrm{mg}$

\section{References}

[1] C. H. Ansel, Pengantar Bentuk Sediaan Farmasi Terjemahan Farida Ibrahim Edisi IV. Jakarta: Universitas Indonesia Press, 1989.

[2] Zubaedah, "Perbandingan Mutu Fisik dan Profil Disolusi Tablet Ibuprofen Merk Dagang dan Generik," Universitas Muhammadiyah Surakarta, 2009.

[3] G. Tiwari et al., "Drug delivery systems: An updated review," Int. J. Pharm. Investig., vol. 2, no. 1, p. 2, (2012), doi: 10.4103/2230$973 \times .96920$. 
[4] K. H. K. Brahma N.Singh, "Floating drug delivery systems: an approach to oral controlled drug delivery via gastric retention," J. Control Release, vol. 63, no. 3, pp. 235-259, (2000), [Online]. Available: https://doi.org/10.1016/S0168-3659(99)002047.

[5] C. G. Wilson and K. Kelly, "Gastrointestinal transit and drug absorption," Pharm. Dissolution Test., vol. 25, no. February, pp. 97125, (2005), doi: 10.3109/9781420077346-4.

[6] N. S. . Gopinath, Harish, Rudru S., Chakravarthi V., Asma S., "Formulation And Evaluation Of Ofloxacin Floating Tablets By Using Hydroxyl Propyl Methyl Cellulose As Polymer," J. Chem. Pharm. Sci., vol. 5, pp. 144-149, (2012).

[7] B. R. Streubel A, Siepmann J, "Multiple unit gastroretentive drug delivery systems: a new preparation method for low density microparticles. J Microencapsul.," J. Microencapsul, vol. 20, no. 3, pp. 329-347, (2003), doi: 10.1080/0265204021000058384.

[8] A. S. Surana and R. K. Kotecha, "An overview on various approaches to oral controlled drug delivery system via gastroretention," Int. J. Pharm. Sci. Rev. Res., vol. 2, no. 2, pp. 68-72, (2010).

[9] R. Khan, "Gastroretentive drug delivery system - A review," Int. J. Pharma Bio Sci., vol. 4, no. 2, pp. 80-89, (2013), doi: 10.21276/apjhs.2014.1.2.9.

[10] M. Cazzola et al., "Efficacy and safety profile of xanthines in COPD: A network metaanalysis," Eur. Respir. Rev., vol. 27, no. 148, pp. 1-12, (2018), doi: 10.1183/16000617.00102018.

[11] A. M. Bayomi, M.A., Al-Suwayeh, S.A., ElHelw, "Excipient-Excipient Interaction in the Design of Sustained-Release Theophylline Tablets: In Vitro and In Vivo Evaluation," Drug Dev. Ind. Pharmac, vol. 27, no. 6, pp. 499-506, (2001).

[12] M. Sowjanya, S. Debnath, P. Lavanya, R. Thejovathi, and M. N. Babu, "Polymers used in the Designing of Controlled Drug Delivery System," Res. J. Pharm. Technol., vol. 10, no. 3 , p. 903, (2017), doi: 10.5958/0974360x.2017.00168.8.

[13] S. Goyal, G. Agarwal, S. Agarwal, and P. K. Karar, "Oral Sustained Release Tablets: An Overview with a special emphasis on Matrix Tablet," Am. J. Adv. Drug Deliv., vol. 05, no. 02, (2017), doi: 10.21767/2321-547x.1000013.

[14] G. T. Reilly, T. M., Tiwari, S. B. dan Krayz, "Formulating Hydrophilic Matrix System," Drug Deliv. Technol., vol. 7, no. 1, pp. 23-27, (2009).

[15] P. Franco and I. De Marco, "The use of poly(Nvinyl pyrrolidone) in the delivery of drugs: A review," Polymers (Basel)., vol. 12, no. 5, pp. 18-21,(2020), doi: 10.3390/POLYM12051114. 